\title{
Evaluación del sindrome isquemia-reperfusión y papel de la preservación del injerto en la extracción renal laparoscópica versus abierta en un modelo experimental porcino de donante vivo
}

\author{
Subirá Ríos D*, Rodríguez Martínez D*, Dulín E**, García Bordas J**, Álvarez Fernández E***, \\ del Cañizo López JF*, Hernández Fernández C*, Lledó García E*.
}

*Servicios de Urología, Medicina y Cirugía Experimental, **Bioquimica y ***Anatomía Patológica. Hospital General Universitario Gregorio Marañón. Madrid.

Actas Urol Esp. 2008;32(1):119-127

\section{RESUMEN}

EVALUACIÓN DEL SÍNDROME ISQUEMIA-REPERFUSIÓN Y PAPEL DE LA PRESERVACIÓN DEL INJERTO EN LA EXTRACCIÓN RENAL LAPAROSCÓPICA VERSUS ABIERTA EN UN MODELO EXPERIMENTAL PORCINO DE DONANTE VIVO

Introducción: La disfunción inicial del injerto renal extraído por laparoscopia en un donante vivo es un aspecto que, aún hoy, crea ciertas controversias. El sindrome de isquemia-reperfusión explicaría parcialmente esta disfunción inicial del injerto trasplantado que, según su intensidad, puede llegar a condicionar una menor supervivencia del riñón. Analizamos en un modelo experimental de donante vivo la repercusión hemodinámica, bioquímica e histológica de la extracción laparoscópica del injerto. Como objetivo secundario evaluamos el papel de la aplicación de una técnica de preservación (perfusión en bomba) durante los minutos de isquemia fría.

Material y metodos: Utilizamos 20 cerdos tipo large-white (peso 40-45 kgrs), que fueron divididos en 4 grupos:

Grupo A: Extracción laparoscópica + perfusión inmediata del injerto tras la extracción mediante bomba pulsátil + Autotrasplante

Grupo B: Extracción laparoscópica + perfusión por gravedad del injerto + Autotrasplante

Grupo C: Extracción por cirugia abierta + perfusión inmediata del injerto tras la extracción mediante bomba pulsátil + Autotrasplante

Grupo D: Extracción por cirugía abierta + perfusión por gravedad del injerto + Autotrasplante

Las extracciones laparoscópica y por cirugia abierta se realizaron por vía transperitoneal con técnica estandarizada y sistemática. La perfusión hipotérmica en bomba se llevó a cabo mediante un sistema pulsátil diseñado por nuestro grupo.

Resultados. Comprobamos que el flujo arterial del riñón trasplantado que previamente fue extraído por laparoscopia, es menor que los extraídos por cirugía abierta, y existe una tendencia a recuperarse tras la primera hora post-reperfusión. La aplicación de un periodo corto de preservación del injerto durante la isquemia fría no mejora el patrón hemodinámico del injerto trasplantado, incluso resulta perjudicial. Por otro lado, la concentración de óxido nítrico en sangre venosa del injerto extraído por laparoscopia fue menor que la de los obtenidos mediante cirugia abierta. Finalmente, el estudio histológico mostró peor conservación de los elementos túbulo-glomerular y endotelial en los órganos extraídos por laparoscopia. Conclusiones: En nuestra experiencia:

- La extracción laparoscópica renal en un modelo de donante vivo frente a la extracción abierta, determina un menor flujo renal y mayor resistencia vascular.

- Esta alteración hemodinámica de los riñones extraídos por laparoscopia tiene tendencia a corregirse a los 60 minutos tras la reperfusión.

- Existe una menor concentración sanguínea de NO en los injertos trasplantados que fueron extraídos por laparoscopia en comparación con el grupo extraídos por cirugía abierta.

Palabras clave: Isquemia-reperfusión. Laparoscopia. Trasplante renal.

\section{ABSTRACT}

ISCHEMIA-REPERFUSION SYNDROME AND ROLE OF PRESERVATION GRAFT TECHNIQUE AFTER LAPAROSCOPIC VERSUS OPEN NEPHRECTOMY IN A EXPERIMENTAL MODEL OF LIVING DONOR KIDNEY TRANSPLANT

Introduction: Delayed graft function alter living donor transplantation is a subject of debate. Delayed graft function can be partially explained by renal ischemia-reperfusion injury, when severe is associated with decreased graft survival. In this experimental living donor model study, we analyze the hemodynamic, histological and biochemical effects of laparoscopic nephrectomy. We also, analyze the effect of a pulsatile machine perfusion for kidney preservation during cold ischemia time.

Material and methods: Twenty large-white pigs (average weight 40-45 kgrs) were divided in 4 experimental groups:

Group A: Laparoscopic nephrectomy+ immediate graft perfusion in pulsatile vacuum pump+autotransplant

Group B: Laparoscopic nephrectomy+ immediate graft perfusion by gravity+autotransplant

Group C: Open nephrectomy+immediate graft perfusion in pulsatile vacuum pump+autotransplant

Group D: Open nephrectomy+ immediate graft perfusion by gravity+autotransplant

Both laparoscopic and open nephrectomy were completed transperitoneally according to standardized technique. Hypothermic perfusion was done in a system designed in our lab.

RESULTS. We observed a decreased renal artery flow in kidneys procured laparoscopically compared to open nephrectomy. We found an artery flow recorvery during the first 60 minutes after revascularization. Renal machine perfusion during cold ischemia time seems to have no beneficial effect, but shows a deleterious effect on hemodynamic event for renal transplantation. Lower plasma nitric oxide level is observed in kidneys obtained by laparoscopy compared with open surgical technique. And finally, we also found higher histological damage in proximal tubular and endothelial cell, in kidneys obtained by laparoscopy compared with open surgery.

Conclusions: In our experience:

- Laparoscopic nephrectomy versus open nephrectomy produces, in a model of living donor transplant, a lower value or renal blood flow and a hig her value of renal vascular resistanse.

- These hemodynamic findings tend to normalize by 60 min after the reperfusion.

A lower blood concentration of nitric oxide after the transplant was detected in laparoscopic group Vs open surgery group. 
$\mathrm{L}$ a nefrectomía laparoscópica de donante vivo, descrita en 1995 por Ratner, ha permitido el desarrollo de los programas de trasplante de donante vivo, aumentando significativamente el número de trasplante renales en los últimos años ${ }^{1}$.

La extracción renal laparoscópica aporta ventajas de morbilidad perioperatoria para el donante: menor necesidad analgésica, menor estancia hospitalaria con una más pronta recuperación postoperatoria ${ }^{2}$. Este cambio en la estrategia produce beneficios en el donante frente a la cirugía abierta clásica.

Pocos son los estudios realizados en humanos acerca de los efectos de la extracción renal laparoscópica en el trasplante renal, la mayoría son estudios retrospectivos ${ }^{3-5}$. Todas las series publicadas coinciden en el aumento del tiempo de isquemia caliente, si bien, existe controversia en la mayor incidencia de disfunción inicial del injerto en los riñones extraídos por laparoscopia ${ }^{6}$.

No han sido publicados muchos estudios que analicen específicamente la influencia de la técnica quirúrgica (laparoscopia vs cirugía abierta) en el proceso de isquemia-reperfusión del injerto renal. Los estudios experimentales en modelos animales grandes ${ }^{7}$ y pequeños ${ }^{8}$, confirman el efecto perjudicial del neumoperitoneo con CO2 sobre el flujo vascular renal determinando oliguria y necrosis tubular aguda ${ }^{9}$.

La disfunción inicial del injerto (DGF), se relaciona directamente con el sindrome isquemiareperfusión (SIR) condicionado por factores conocidos como el tiempo de preservación del injerto, la presión intrabdominal del neumoperitoneo y otros, menos conocidos como factores inmunológicos.

\section{JUSTIFICACIÓN DEL TRABAJO Y OBJETIVOS}

Debido a la disparidad de resultados tanto en los estudios experimentales como en clínica en cuanto al efecto pernicioso de la extracción laparoscópica sobre el injerto renal, nos planteamos estudiar en un modelo experimental de animal completo de gran tamaño, como modelo más exacto posible al donante vivo en clínica, el efecto de la técnica quirúrgica de obtención del injerto renal, mediante evaluación de parámetros:
1) Hemodinámicos: flujo vascular renal del riñón trasplantado en el periodo inmediato de revascularización - resistencia vascular renal (indicadores directos del síndrome isquemia repercusión)

2) Bioquímicos: óxido nítrico (NO) conocido mediador del síndrome isquemia-reperfusión (indicador indirecto del funcionalismo endotelial)

3) Histológicos: evaluación de las consecuencias estructurales del proceso mediante microscopia electrónica.

\section{MATERIAL-MÉTODOS. DISEÑO EXPERIMENTAL}

Los factores de nuestro estudio fueron 2: técnica quirúrgica de extracción y aplicación de perfusión de órgano aislado en bomba previamente al implante. Los niveles aplicados de cada factor previamente al trasplante fueron dos: extracción renal laparoscópica Versus cirugía abierta y preservación mediante perfusión de órgano aislado en bomba regulada por vacío durante $60 \mathrm{~min}$ con solución de perfusión de Belzer Versus perfusión por gravedad con solución de Viaspán-UW.

Las variables analizadas fueron: flujo vascular renal (FVR), presión arterial sistémica (PAS), resistencia vascular renal (RVR) y óxido nítrico (ON). Establecimos cuatro tratamientos por combinación de los distintos niveles de nuestros factores, definidos por los grupos:

Grupo A: Extracción laparoscópica + perfusión inmediata del injerto tras la extracción mediante bomba pulsátil dos grupos + Trasplante

Grupo B: Extracción laparoscópica + perfusión por gravedad del injerto + Trasplante

Grupo C: Extracción por cirugía abierta + perfusión inmediata del injerto tras la extracción mediante bomba pulsátil dos grupos + Trasplante

Grupo D: Extracción por cirugía abierta + perfusión por gravedad del injerto + Trasplante

Utilizamos en cada grupo 5 animales de experimentación homogéneos, cerdos large-white (peso 40-45 kg). La extracción laparoscópica se realizó por vía transperitoneal con técnica estandarizada y sistemática:

- Tres puertos de entrada para la colocación de trócares.

- Neumoperitoneo con CO2 fijando como límite máximo de presión intrabdominal $10 \mathrm{mmHg}$. 
- En todos los casos el tiempo de isquemia caliente fue menor de 5 minutos.

El manejo de los animales se ha realizado siguiendo la normativa legal según el Real Decreto 223/1988, de 14 de marzo (R. 601): Animales. Protección de los utilizados para experimentación y otros fines científicos (Ministerio de Agricultura, Pesca y Alimentación), que incorpora las disposiciones legales, reglamentarias y administrativas de los estados miembros de la CEE respecto a la protección de los animales utilizados para experimentación y otros fines científicos, de la Directiva del Consejo 86/609/CEE (Leg. CC. EE. 4390) aprobada el 24 de noviembre de 1996. Todos los animales fueron hidratados con suero salino fisiológico (1500 ml) y osmofundina (manitol al 20\%, osmola-

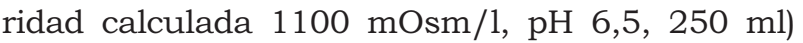
desde el inicio de la intervención hasta la extracción de los riñones. Durante todo el procedimiento quirúrgico se realizó perfusión continua en bomba de Heparina al 1\%.

La extracción abierta de los injertos fue vía abdominal. En todos los grupos se procedió al clampaje del riñón no extraído. La reposición de liquidos durante la extracción fue mediante suero glucosalino a velocidad de $15 \mathrm{mil} / \mathrm{min}$.

Se procedió al autoimplante del injerto al finalizar el tiempo de perfusión renal, mediante anastomosis a aorta y cava con tiempo de isquemia de revascularización similar en todos los grupos.

Las determinaciones de NO se realizaron mediante la extracción sanguínea de la vena renal del injerto cada 15 minutos durante dos horas. Los parámetros hemodinámicos se registraron en tiempo real el flujo arterial renal durante 120 minutos . Se utilizó un flujómetro ultrasónico T-109 (Transonic Systems Inc). La P.P.R. se determinó mediante un transductor desechable (Transpac L978-39 Abbott CCS) ubicado por vía percutánea carotídea junto al ostium de salida de la arteria. La medida de resistencia vascular renal (RVR) fue realizada por el sistema, según el cociente P.P.R./F.P.R. ( $\mathrm{mmHg} / \mathrm{ml} / \mathrm{mto}$ ).

Se tomaron muestras de sangre de vena renal para determinaciones de óxido nítrico a $0^{-}, 1^{\circ}$, $30^{\circ}, 4^{\circ}, 60$ min tras el desclampaje. El NO producido fue determinado con un ensayo espectrofotométrico, mediante la medición de los productos acumulados de degradación estables, nitritos y nitratos. Para monitorizar ambos llevamos a cabo la conversión enzimática de nitratos en nitritos mediante la nitrato reductasa, seguida por la cuantificación de nitritos mediante el reactante de Griess. Previamente a iniciar el ensayo, las muestras que contenian altos niveles proteicos fueron filtradas, para evitar un precipitado que interfiriese con la medición. Se preparó una curva estándar en un rango de 0-100 $\mu \mathrm{M}$ de NO.

Se realizaron estudios microscópicos con microscopía convencional y electrónica en todos los casos tras el procedimiento. La tinción de hematoxilina-eosina se usó en la microscopía convencional; la técnica de Reynolds (tinción ultraestructural de acetato de uranilo y citrato plúmbico) para la microscopía electrónica (JEOLJEM 100 SXR).

\section{RESULTADOS}

1. Análisis del síndrome isquemia-reperfusión. Estudio comparativo entre grupo extracción laparoscópica y abierta: flujo arterial y resistencia vascular

2. Determinación de NO como mediador del Sindrome de isquemia-reperfusión

3. Análisis del papel del tipo de perfusión en el flujo arterial y resistencia arterial: bomba pulsátil vs gravedad.

4. Estudio anatomopatológico de los riñones según tipo de extracción.

El análisis del síndrome isquemia-reperfusión en la extracción renal laparoscópica, se realizó mediante la determinación del flujo sanguíneo renal, la resistencia vascular renal y niveles de NO tras la revascularización del injerto al finalizar el autotrasplante (Tablas 1 y 2).

Las Figuras 1 y 2 muestran las modificaciones del flujo renal en tiempo real durante las dos primeras horas tras la reperfusión (la Figura 2 muestra los valores de flujo corregido por peso del riñón). Se observa comparativamente en el grupo de extracción laparascópica un menor flujo arterial frente al grupo de extracción abierta durante los primeros 60 minutos (flujo arterial medio corregido por peso del riñón $107 \mathrm{ml} / \mathrm{min}$ y $120 \mathrm{ml} / \mathrm{min}$ en los grupos laparoscópicos frente a 146 y $208 \mathrm{ml} / \mathrm{min}$ en el grupo de extracción abierta). Posteriormente existe una tendencia de aumento del flujo arterial en el grupo de extrac- 
Tabla 1. Valores medios de P.A.S., F.V.R., R.V.R. y F.V.R./100 g peso según el grupo de estudio

\begin{tabular}{lcccc}
\hline Grupo & $\begin{array}{c}\text { Presion arterial } \\
\text { sistémica (mmH) }\end{array}$ & $\begin{array}{c}\text { Flujo vascular } \\
\text { renal (ml/min) }\end{array}$ & $\begin{array}{c}\text { Resistencia vascular } \\
\text { renal (mmHg/ml/min) }\end{array}$ & $\begin{array}{c}\text { Flujo vascular } \\
\text { renal/ 100 g peso } \\
\text { (ml/min/100 g }\end{array}$ \\
\hline Cirugia abierta-perfusion & 88,04 & 125,23 & 0,85 & 146,07 \\
Cirugia abierta-no perfusión & 106,34 & 136,84 & 0,67 & 208,85 \\
Laparoscopia-no perfusión & 88,17 & 12,08 & 0,85 & 120,13 \\
Laparoscopia-perfusión & 94,34 & 88,37 & 1,11 & 107,63
\end{tabular}

Tabla 2. Concentración media de NO según grupo de estudio

\begin{tabular}{lccc}
\hline $\begin{array}{l}\text { Tiempo } \\
\text { min }\end{array}$ & $\begin{array}{c}\text { Laparoscopia } \\
\text { con perfusión } \\
\text { Media [NO] mm }\end{array}$ & $\begin{array}{c}\text { Laparoscopia } \\
\text { sin perfusión } \\
\text { Media [NO] mm }\end{array}$ & $\begin{array}{c}\text { Cirugia } \\
\text { abierta } \\
\text { Media [NO] mm }\end{array}$ \\
\hline 0 & 12,89 & 12,78 & 31,75 \\
15 & 18,85 & 16,30 & 23,80 \\
30 & 17,15 & 10,89 & 23,85 \\
45 & 17,65 & 18,10 & 21,40 \\
60 & 14,28 & 10,30 & 34,70 \\
75 & 14,20 & 15,55 & 26,65 \\
90 & 10,65 & 16,70 & 34,55 \\
105 & 13,05 & 22,40 & 22,05 \\
120 & 15,65 & 21,70 & 37,80 \\
Media & 14,93 & 16,08 & 28,50 \\
\hline
\end{tabular}

ción laparoscópica hasta igualarse en los siguientes 60 minutos de reperfusión con el grupo de extracción abierta. Además se observa que, en ambos grupos de extracción, aquellos que fueron perfundidos mediante el sistema de bomba pulsátil tenían un flujo arterial renal menor que los que fueron lavados por gravedad, $107 \mathrm{ml} / \mathrm{min}$ frente a $120 \mathrm{ml} / \mathrm{min}$ entre los riñones extraídos por laparoscopia y un flujo de $146 \mathrm{ml} / \mathrm{min}$ frente a $208 \mathrm{ml} / \mathrm{min}$ en los extraídos por cirugía abierta.

El análisis de la resistencia vascular renal, muestra así mismo, una mayor resistencia en el grupo de extracción laparoscópica, media de 0,85 frente a 0,67 $(\mathrm{p}<0,05)$ en el grupo de cirugía abierta, con tendencia tras los primeros 60 minutos a igualarse con el grupo de extracción abierta (Fig. 3).

De igual modo, si analizamos los grupos según el modo de preservación, encontramos una mayor resistencia vascular en aquellos riñones que fueron sometidos a perfusión en bomba independientemente del grupo de extracción que se trate.
Por último la determinación de NO, cada 15 minutos durante 120 minutos tras el desclampaje renal, muestra una mayor concentración media de óxido nítrico en sangre en el grupo de extracción renal abierta frente al grupo de cirugía laparoscópica (Fig. 4). La concentración media de NO a los 120 minutos de revascularización del riñón trasplantado fue de $15 \mu \mathrm{m}$ en el grupo de extracción laparoscópica sin perfusión, 21 $\mu \mathrm{m}$ en el grupo laparoscópico con perfusión en bomba y de $37 \mu \mathrm{m}$ en el grupo de extracción abierta $(p<0,05)$. Se observa una tendencia a aumentar los niveles de NO con el tiempo tras la revascularización del riñón, sin embargo los niveles de $\mathrm{NO}$ en el grupo de extracción abierta se mantienen estables a lo largo de los 120 minutos. Parece que los riñones que fueron preservados mediante perfusión con bomba durante el tiempo de isquemia fría, produjeron menor concentración de NO.

El estudio anatomopatológico tanto por microscopía óptica como electrónica, muestran una mayor desestructuración celular con aumento de vacuolas citosplasmáticas y roturas de membranas, así como mayor grado de retracción glomerular y edema tubular en aquellos riñones que fueron extraídos por laparoscopia frente a los extraídos por cirugía abierta. Además, estas alteraciones celulares son más acusadas en los riñones que fueron perfundidos mediante bomba pulsátil, independientemente del modo de extracción realizada. El estudio mediante microscopía electrónica del túbulo y endotelio mostró lesiones endoteliales significativas y ocupación de la luz microvascular 


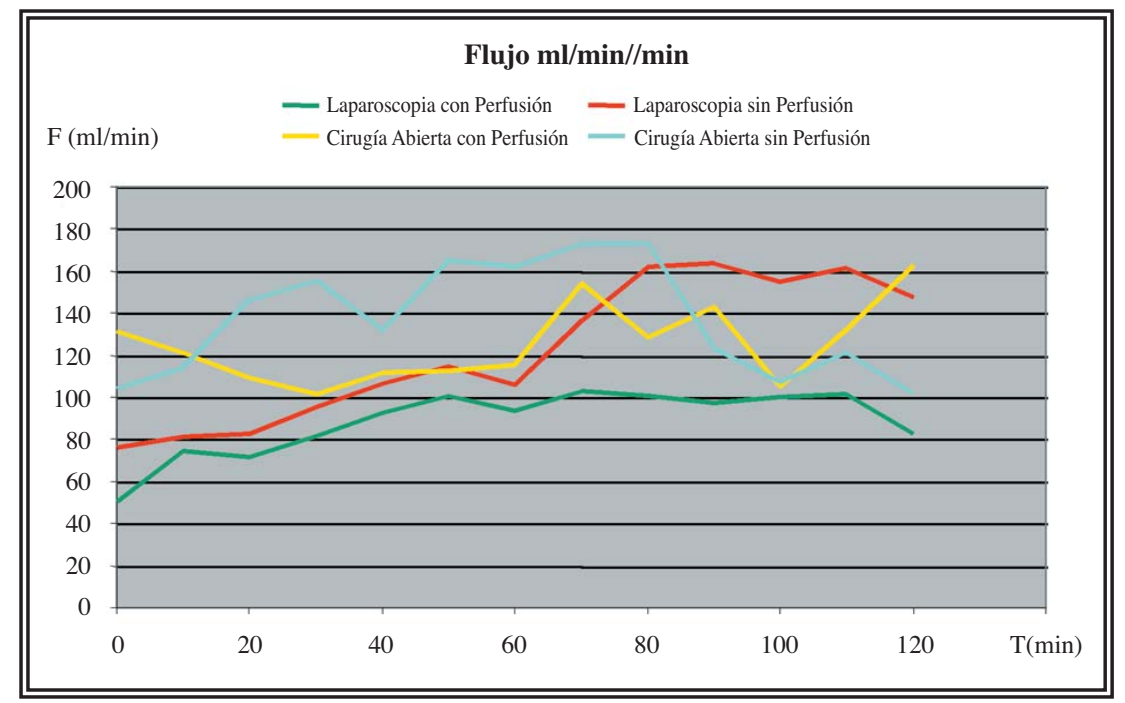

FIGURA 1. Flujo renal tras repercusión según grupo estudio.

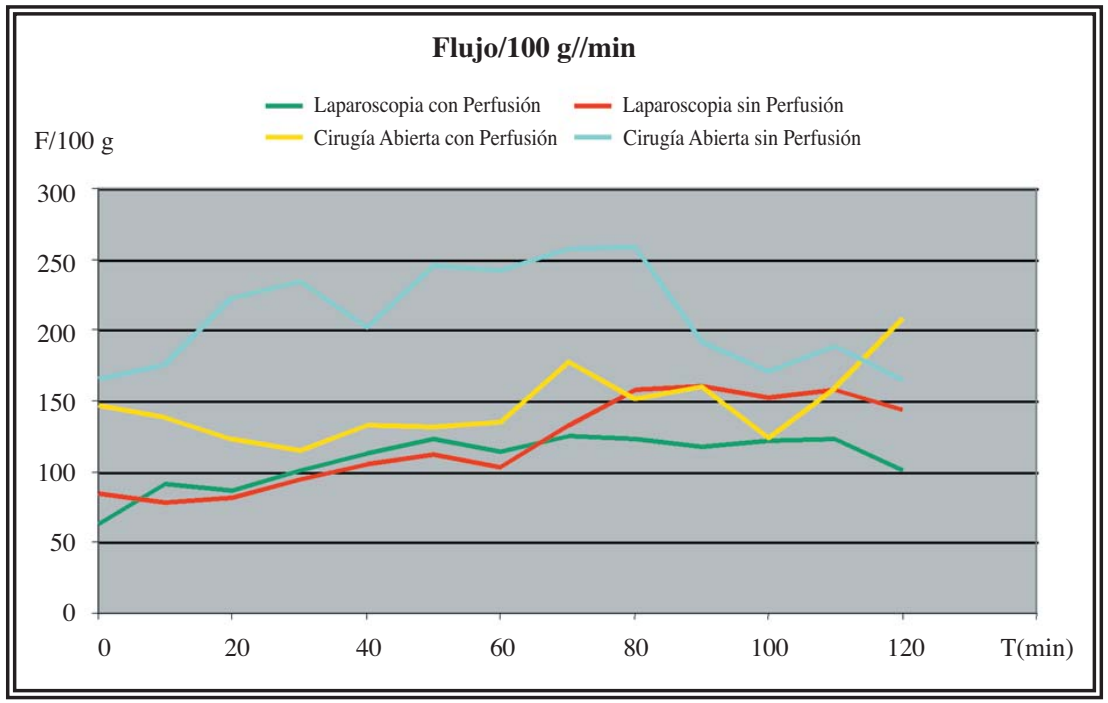

FIGURA 2. Flujo corregido por peso del injerto por grupo de estudio.

El síndrome isquemia-reperfusión al que está sometido el injerto durante su extracción y posterior revascularización contribuye a explicar la fisiopatología de la disfunción inicial del riñón trasplantado. El flujo sanguíneo renal tras la reperfusión del injerto trasplantado, refleja el grado de este síndrome: cuanto mayor es el flujo renal menor es el sindrome de isquemia-reperfusión y por tanto mejor función renal ${ }^{11}$. La extracción laparoscópica renal en comparación con la extracción mediante cirugía abierta, condiciona una disminución significativa del flujo sanguíneo post-trasplante ${ }^{12}$. Varios son los factores identificados en el síndrome isquemiareperfusión: factores directos como los tiempos de isquemia (caliente, fria), y otros menos conocidos como los inmunológicos que modularian la respuesta inmune del injerto hacia un mayor riesgo de rechazo. El NO, la endotelina 1, la IL 2 y 6 , actuarian como mediadores de este sindrome ${ }^{13}$.

$\mathrm{El}$ endotelio vascular del injerto desempeña un papel fundamental en el tono vascular, y por tanto en su flujo. Esta función reguladora del endotelio la realiza mediante la liberación de sustancias vasoactivas entre ellas la endotelina - 1

por microtrombos de fibrina, especialmente en los grupos de órganos extraídos por laparoscopia (Figs. 5 y 6).

\section{DISCUSIÓN}

La disfunción inicial del injerto renal trasplantado procedente de donante vivo se ha cifrado entre el 5\% y el 10\%, independientemente de la técnica de extracción. Esta disfunción, definida por la necesidad de diálisis durante la primera semana post-trasplante, parece que se asocia a un mayor riesgo de rechazo agudo y crónico que condicionaría una menor supervivencia del injerto ${ }^{10}$. y el óxido nítrico (NO). La endotelina -1, conocido vasoconstrictor, está implicado en el aumento de las resistencias vasculares renales, disminuyendo el flujo sanguíneo renal. Su liberación aumenta durante las fases de isquemia renal tanto fría como caliente. La endotelina -1, está involucrado en el rechazo agudo y crónico del injerto renal trasplantado ${ }^{14}$. Además se ha demostrado su papel mediador potenciador del sindrome isquemia-repercusión ${ }^{15}$. Por otra parte, el NO es un potente vasodilatador que procede de la transformación de la 1-arginina por la óxido nítrico sintetasa. 


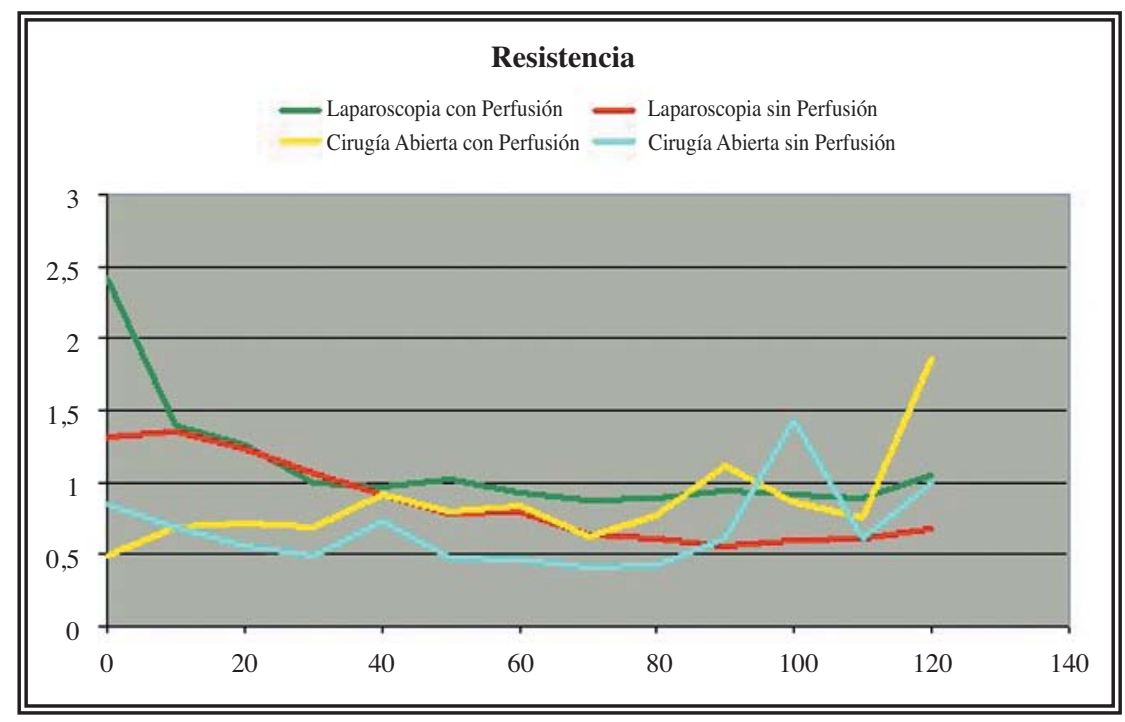

FIGURA 3. Resistencia vascular $(\mathrm{mmHg} / \mathrm{ml} / \mathrm{min})$ tras revascularización del injerto según grupo estudio.

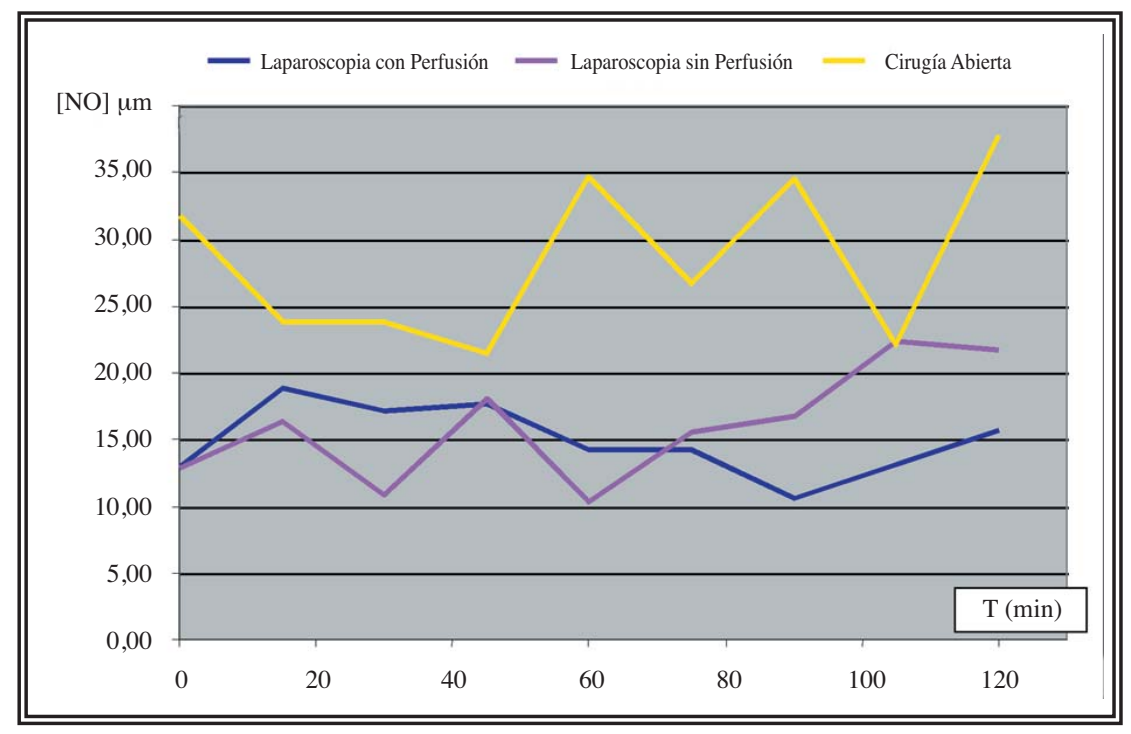

FIGURA 4. [NO] Mm tras revascularización por grupo estudio.

Las concentraciones de estos mediadores, endotelina -1 y NO, producidos por el endotelio vascular, son esenciales para el control del la perfusión del injerto. Jeong et al. ${ }^{16}$, en un estudio experimental, modulan el efecto del síndrome isquemia-reperfusión mediante el uso de dadores de NO, nitroprusiato, durante la fase previa a la isquemia caliente de la extracción renal, demostrando que el uso de precursores de $\mathrm{NO}$ en fases precoces a la isquemia del injerto extraído, se asocia una mejor función del injerto y, éste se asocia a la supresión en la liberación de endotelina-1.
El papel de las citocinas como indicadores de agresividad quirúrgica, ha sido ampliamente publicado. Fornara et al. ${ }^{17}$. En un modelo experimental con cerdos, evidencian un aumento de los niveles de citocinas, asociado a una mayor agresividad quirúrgica, en los animales sometidos a nefrectomía abierta frente a los sometidos a nefrectomía laparoscópica. De igual manera Burgos et al. ${ }^{13}$ en otro estudio experimental con cerdos, evidencian un mayor aumento de citocinas (TNF,IL-2,IL-6 y IL-10) en la nefrectomía abierta frente a la nefrectomía laparoscópica y además asocian el aumento de IL-10 durante el trasplante renal a una mejor función del injerto, frente a niveles elevados de IL-2 durante la extracción que parece asociarse a una peor función del injerto trasplantado.

La incidencia de mayor disfunción inicial del injerto en donantes vivos es controvertida. Nogueira ${ }^{18}$ encuentra en una serie retrospectiva de 143 riñones trasplantados extraídos por laparoscopia frente a 100 riñones extraídos por cirugía abierta, una incidencia mayor de DFG en los riñones extraídos por laparoscopia que por vía abierta, $7,6 \%$ vs $2,0 \%$ respectivamente. Si bien, estos autores observaron que esta disfunción era transitoria. Tras una diferencia inicial en la función renal a la semana del trasplante, a favor de los riñones que fueron extraídos por cirugía abierta, ésta, se corregía a los 3 meses post-trasplante. Por otra parte, recientes estudios confirman que la supervivencia del injerto extraído por laparoscopia al año del trasplante, es similar a los extraídos por cirugia abierta ${ }^{19,20}$. Probablemente esta disparidad de datos publicados en la literatura, tanto en revisiones retrospectivas clínicas como en estudios experimentales sean debidas a las diferentes 


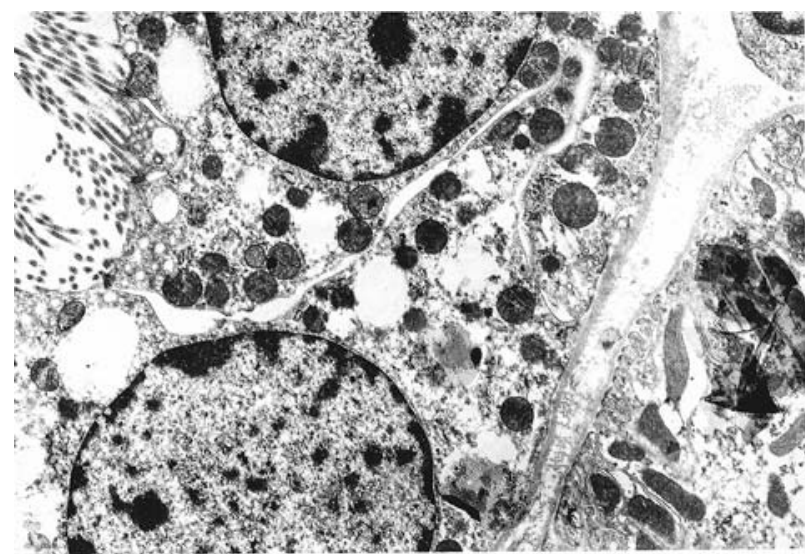

FIGURA 5. Vacualización citoplasmática. Desestructuración del túbulo. Microscopía electrónica. Injerto extraido por laparoscopia.

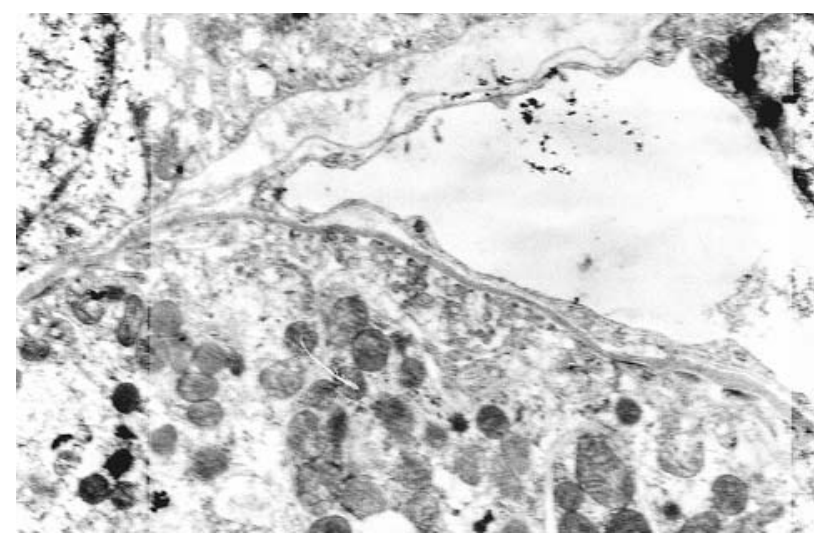

FIGURA 6. Alteraciones del endotelio. Microscopía electrónica. Injerto renal extraido por laparoscopia.

condiciones en la que la extracción renal por cirugía laparoscópica fue realizada. En algunos casos de estudios experimentales, no reproducen fielmente las condiciones de extracción renal laparoscópica habituales en clínica. Derweesh et al. ${ }^{21}$ en un estudio retrospectivo de trasplante de donante vivo, de 101 riñones extraídos por laparoscopia frente a 35 extraídos por cirugía abierta, no encuentran una mayor incidencia de disfunción inicial del injerto extraído por laparoscopia, a diferencia de lo publicado por otros autores ${ }^{22}$. Derweesh hace énfasis en las condiciones técnicas de la extracción laparoscópica para conseguir estos datos funcionales.

Múltiples estudios han demostrado que el neumoperitoneo prolongado determina oliguria, disminución del flujo sanguíneo renal y del filtrado gromerular $^{23}$. Estos efectos del neumoperitoneo sobre la fisiopatología del riñón es multifactorial: por una parte por el efecto mecánico de compresión sobre la vena renal y el parénquima, por otra parte por la liberación de vasoconstrictores que determinarian un menor filtrado glomerular, además el neumoperitoneo condiciona una mayor susceptibilidad al rechazo ${ }^{24,25}$. Aunque la disfunción del riñón durante la extracción laparoscópica es transitoria ${ }^{26}$, también pueden condicionar una mayor predisposición a una disfunción permanente del injerto trasplantado ${ }^{27}$. Por eso parece crucial el manejo intraoperatorio de la extracción renal laparoscópica y ello, también, explicaría la disparidad de resultados de disfunción del riñón trasplantado publicados en la literatura.

Los datos contradictorios publicados en cuanto a la mayor incidencia de disfunción inicial del injerto extraído por laparoscopia nos han animado a profundizar en el estudio del flujo arterial tras la reperfusión como principal indicador del síndrome isquemia-reperfusión (SIR). Puesto que es sabido que el grado de afectación del sindrome isquemia-reperfusión puede condicionar desde una disfunción inicial leve del injerto trasplantado hasta, en mayor grado, una disfunción que condicione una limitación de la supervivencia del injerto.

Hemos realizado un modelo de donante vivo, con el cerdo como animal de experimentación, intentando reproducir los factores que se dan en clínica. Un neumoperitoneo con PIA (presión intrabdominal) $>10 \mathrm{mmHg}$ se ha demostrado que produce una disminución del flujo arterial, filtrado glomerular y diuresis, por lo que en nuestro modelo hemos trabajado con PIA máxima de $10 \mathrm{mmHg}$, similar al que se emplea en clínica ${ }^{28}$. También se ha comprobado que tiempos de isquemia caliente $>10$ minutos determinan un sindrome de isquemia-reperfusión más severo, por lo que en nuestro modelo nuestro tiempo de isquemia caliente en la extracción laparoscópica ha sido siempre inferior a 5 minutos, reproduciendo también la media de lo publicado en series de nefrectomía de donante vivo $6,21,29,30$.

En nuestro estudio, hemos objetivado una mayor resistencia vascular y menor flujo arterial en el grupo de riñones extraídos por laparoscopia tras la reperfusión del injerto, con una tendencia a igualarse con el grupo control, extracción abier- 
ta, tras 60 minutos de reperfusión. Esto explicaría la disfunción del injerto limitada en el tiempo según distintos autores entre 1 a 3 meses posttrasplante ${ }^{31}$. Estos datos hemodinámicos de nuestro estudio se ven apoyados por las alteraciones histológicas encontradas en el estudio anatomopatológico realizado de los riñones de cada grupo de nuestro estudio. Es decir, aquellos riñones sometidos a extracción laparoscópica y aquellos riñones sometidos a preservación mediante bomba pulsátil independientemente del modo de extracción, presentaban un peor patrón de revascularización, medido por el flujo arterialresistencia vascular, y a nivel histológico una mayor desestructuración glomerular, así como de los túbulos y endotelio vascular.

Los niveles más bajos de NO parecen relacionarse con un menor flujo arterial y mayor resistencia en los injertos extraídos por laparoscopia. Estos hechos pueden sugerir un cierto grado de disfunción endotelial en los órganos extraídos por laparoscopia, y confirmarian el papel importante que el NO tiene como molécula reguladora del estado de contracción vascular durante el SIR, de acuerdo con lo publicado ${ }^{32}$. Posiblemente, la modulación del NO presente durante la revascularización del injerto mediante dadores de NO puedan modificar el SIR, así como otros factores como la utilización de vasodilatadores locales de la arteria renal que reduzcan el riesgo de vasoespasmo inducido por la mayor manipulación de los vasos durante la extracción laparoscópica y el incremento de aporte de líquidos intravascular para minimizar la disminución de flujo vascular inducido por el neumoperitoneo que condicionaría oliguria y disminución del filtrado gromerular ${ }^{21}$.

Nuestros datos confirman la disminución del flujo sanguíneo renal en los riñones extraídos por laparoscopia pero, posiblemente al limitar los factores que lo condicionan, este flujo tiene tendencia ha aumentar a partir de los 60 minutos de la revascularización. La corrección de estos factores descritos mediante el uso de papaverina tópica, dopamina, PIA $<12 \mathrm{mmHg}$, tiempos de isquemia caliente $<5$ minutos, aumento de aportes de fluidos intravasculares y, la experiencia quirúrgica laparoscópica, posiblemente sean determinantes en la ausencia de disfunción inicial del injerto en series clínicas de otros autores publicadas ${ }^{6}$.
Por último, la utilización durante la perfusión del injerto de bomba pulsátil en nuestro estudio resulta perjudicial, reflejándose tanto en el grupo de extracción laparoscópica como en el control de cirugia abierta, una disminución del flujo arterial renal del injerto asociada a una mayor resistencia vascular del injerto trasplantado. Este efecto podría explicarse por el daño sobre endotelio que supondría el bombeo pulsátil del líquido de perfusión. Estos datos están en contraposición con el uso de bomba de perfusión en riñones procedentes de donante cadáver durante el tiempo de isquemia fría que parece podrían mejorar los resultados funcionales del injerto ${ }^{32}$. Posiblemente en el trasplante renal de donante vivo, los sistemas de preservación del injerto, durante la fase de isquemia fría, no sólo no son capaces de modificar el síndrome de isquemia-reperfusión asociado a la extracción renal laparoscópica, sino que resulta perjudicial. La reducción del tiempo de isquemia fría, sería el principal factor condicionante en la función del injerto trasplantado ${ }^{6}$.

\section{CONCLUSIONES}

- La extracción laparoscópica renal en un modelo de donante vivo frente a la extracción abierta, determina un menor flujo renal y mayor resistencia vascular.

Ésta alteración hemodinámica de los riñones extraídos por laparoscopia tiene tendencia a corregirse a los 60 minutos tras la reperfusión.

- Existe una menor concentración sanguínea de $\mathrm{NO}$ en los injertos trasplantados que fueron extraídos por laparoscopia en comparación con el grupo extraídos por cirugía abierta.

- Independientemente del tipo de extracción renal en un modelo de donante vivo, el uso del sistema de bomba pulsátil frente a la perfusión por gravedad durante la fase de perfusión renal, isquemia fría, se asocia a una disminución del flujo arterial renal y una mayor resistencia vascular, con la consiguiente mayor severidad del SIR.

Anexo: Proyecto financiado con Beca Investigación FIS 03/1130.

\section{REFERENCIAS}

1. Schweitzer EJ, Wilson J, Jacobs S, Machan CH, Philosophe B, Farney A, et al. Increased rates of donation with laparoscopic donor nephrectomy. Ann Surg. 2000;232(3):392-400. 2. Jacobs SC, Cho E, Dunkin BJ, Flowers JC, Schweitzer E, Cangro $\mathrm{C}$, et al. Laparoscopic live donor nephrectomy: the University of Maryland 3-year experience. J Urol 2000;164 (5):1494-1499. 
3. Troppmann C, Ormond DB, Perez RV. Laparoscopic (vs open) live donor nephrectomy : a UNOS database analysis of early graft function and survival. Am J Transplant. 2003;3(10):1295-1301.

4. Troppmann C, McBride MA, Baker TJ, Perez RV. Laparoscopic live donor nephrectomy: arisk factor for delayed function and rejection in pediatric kidney recipients? A UNOS analysis. Am J Transplant. 2005;5(1):175-182.

5. Feifer AH, Fong BC, Feldman L, Fried G, Stein LA, Metrakos P, et al. Preoperative evaluation of laparoscopic living renal donors with computerized tomography and its effect on donor morbidity and graft function. Can J Urol. 2005;12(3): 2713-2721.

6. Abreu SC, Goldfarb DA, Derweesh I, Thornton J, Urbain $\mathrm{JL}$, Mascha E, et al. Factors related to delayed graft function after laparoscopic live donor nephrectomy. J Urol. 2004;171(1):52-57.

7. London E, Ho H, Neuhaus. Effect of intravascular volume expansion on renal function during prolonged $\mathrm{CO} 2$ pneumoperitoneum. Ann Surg. 2000; 231(2):195-201.

8. Kirsch AJ, Hensle TW, Chang DT, Kayton ML, Olsson CA, Sawczuk IS. Renal effects of $\mathrm{CO} 2$ insufflation : Oliguria and acute renal dysfunction in a rat pneumoperitoneum. Urology. 1994;43(4):453-459

9. Chang DT, Kirsch AJ, Sawczuk IS. Oliguria during laparoscopic surgery. J Endourol. 1994;8(5):349-352.

10. Brentan P, Lobo E, Chang JA. Assessment of preservation induced reperfusion injury viaintraoperative renal transplant blood flor and endothelin concentration studies. J Urol. 1997;158:174-181.

11. Matsuno N, Kozaki K, Degawa H, Narumi Y, Suzuki N, Kikuchi K. Importance of machine perfusion in kidney preservation. Transplant Proc. 1999;31(5):2004-2005.

12. Burgos FJ, Pascual J, Briones B. Influence of laparoscopic live donor nephrectomy in ischemia-reperfusion sindrome and renal function alter kidney transplantation: an experimental study. Transplant Proc. 2003;35:1664-1665.

13. Burgos FJ, Linares A, Pascual J, Marcen R, Villafruela J, Zamora $\mathrm{J}$ et al. Modifications of renal blood flow and serum interleukin levels induced by laparoscopic and open living donor nephrectomies for kidney transplant: an experimental study in pigs. Transplant Proc 2005;37(9):36763678.

14. Castillo J, Berrazueta JR, Herrera L, de Vega MJ, Val JF, Amado JA, et al. Nitric oxide and endothelins are effector agents of hyperacurate vascular rejection. Transplant Proc. 1996;28(2):635.

15. Kumano K, Masui N, Soh S, Mashimo S, Endo T. Plasma endothelin level following kidney transplantation. Transplant Proc. 1994;26(4):2114-2116.

16. Jeong GY, Chung KY, Lee WJ, Kim YS, Sung SH. The effect of a nitric oxide donor on endogenous endothelin-1 expression in renal ischemia/reperfusion injury. Transplant Proc 2004;36(7):1943-1945.

17. Fornara P, Doehn C, Seyfarth M, Jocham D. Why is urological laparoscopy minimally invasive? Eur Urol. 2000;37 (3):241-250.

18. Nogueira JM, Cangro CB, Fink JC, Schweitzer E, Wiland A, Klassen DK et al. A comparison of recipient renal outcomes with laparoscopic versus open live donor nephrectomy. Transplantation. 1999;67(5): 722-728.
19. Sellers MT, Gallichio MH, Hudson SL, Young CJ, Bynon JS, Eckhoff DE, et al. Improved outcomes in cadaveric renal allografts with pulsatile preservation. Clin Transplant. 2000; 14(6):543-549.

20. Reddy KS, Mastrangelo MD, Johnston T, Khan T, Waid T, McKeown $\mathrm{W}$ et al. Recipient outcome following living donor kidney transplantation using kidneys procured laparoscopically. Clin transplant. 2003;17(Suppl 9):44-47.

21. Derweesh IH, Goldfarb DA, Abreu SC, Goel M, Flechner SM, Modlin C, et al. Laparoscopic live donor nephrectomy has equivalent early and late renal function outcomes compared with open donor nephrectomy. Urology. 2005; 65(5):862-866

22. Barry JM. Laparoscopic donor nephrectomy. Transplantation. 2000;70(10):1546-1548.

23. Chiu AW, Chang LS, Birkett DH, Babayan RK. The impact of pneumoperitoneum, pneumoretroperitoneum and gasless laparoscopy on the systemic and renal hemodynamics. J Am Coll Surg. 1995;181(5):397-406.

24. Dunn MD, Mc Dougall EM. Renal physiology:laparoscopic considerations. Urol Clin North Am. 2000;27(4):609-614.

25. Ibrahim S, Jacobs F, Zukin Y, Enriquez D, Holt D, Baldwin $\mathrm{W} 3 \mathrm{rd}$, et al. Immunohistochemical manifestations of unilateral kidney isquemia. Clin Transpl. 1996;10(6 Pt 2): 646-652

26. Hazabroek EJ, de Bruin RW, Bouvy ND. Long-term impact of pneumoperitoneum used for laparoscopic donor nephrectomy on renal function and histomorphology in donor and recipient rats. Ann Surg. 2003;237(3):351-357.

27. Bolte SL, Chin LT, Moon TD, D`Alessandro AM, Nakada SY, Becker YT et al. Maintaining urine production and early allograft function during laparoscopic donor nephrectomy. Urology. 2006;68(4):747-750.

28. Harman RK, Kron IL, McLachlan HD. Elevated intrabdominal pressure and renal function. Am Surg. 1982;196: 594-597.

29. Sasaki TM, Finelli F, Bugarin E, Fowlkes D, Trollinger J, Barhyte DY, et al. Islaparoscopic donor nephrectomy the new criterion standard?. Arch Surg. 2000;135(8):943-947.

30. Soulsby RE, Evans LJ, Rigg KM, Shehata M. Warm ischemic time during laparoscopic live donor nephrectomy: effects on graft function. Transplant Proc. 2005;37(2):620622.

31. Ratner LE, Montgomery RA, Maley WR, Cohen C, Burdick J, Chavin KD et al. Laparoscopic live donor nephrectomy: the recipient. Transplantation. 2000; 69(11):2319-2323.

32. Guarrera JV, Polyak MMR, Arrington B, Boykin J, Brown T, Jean-Jacques MA et al. Pushing the envelope in renal preservation: improved results with novel perfusate modifications for pulsatile machine perfusion of cadaver kidneys. Transplant Proc. 2004;36(5):1257-1260.

Correspondencia autor: Dr. D. Subirá Ríos

Servicio Urología

Hospital General Universitario Gregorio Marañón

Doctor Esquerdo, 46 - 28007 Madrid

Tel.: 915868000

E-mail autor: ibydoc71@hotmail.com

Información artículo: Original 